\title{
Iterative Detection of Three-Stage Concatenated FFH-MFSK
}

\author{
Sohail Ahmed, Robert G. Maunder, Lie-Liang Yang and Lajos Hanzo \\ School of ECS, University of Southampton, SO17 1BJ, UK. \\ Tel: +44-23-80593125, Fax: +44-23-80594508 \\ Email: sohailahmed71@ @otmail.com,\{rm02r,lly,lh\}@ecs.soton.ac.uk; http://www-mobile.ecs.soton.ac.uk
}

\begin{abstract}
Serially concatenated and iteratively decoded Irregular Variable Length Coding (IrVLC) combined with precoded Fast Frequency Hopping (FFH) $M$-ary Frequency Shift Keying (MFSK) is considered. We employ EXtrinsic Information Transfer (EXIT) charts to investigate the 3-stage concatenation of the FFH-MFSK demodulator, the rate-1 decoder and the outer IrVLC decoder. The proposed joint source and channel coding scheme is capable of operating at low Signal-to-Noise Ratio (SNR) in Rayleigh fading channels contaminated by Partial Band Noise Jamming (PBNJ). The IrVLC scheme is comprised of a number of component Variable Length Coding (VLC) codebooks employing different coding rates for encoding particular fractions of the input source symbol stream. These fractions may be chosen with the aid of EXIT charts in order to shape the inverted EXIT curve of the IrVLC codec so that it can be matched with the EXIT curve of the inner decoder. We demonstrate that using the proposed scheme an infinitesimally low bit error ratio may be achieved at low SNR values.
\end{abstract}

\section{INTRODUCTION}

Fast Frequency Hopping (FFH) $M$-ary Frequency Shift Keying (MFSK) has been shown to efficiently combat Partial Band Noise Jamming (PBNJ) [1]. By transmitting every symbol using multiple hops, FFH systems benefit from both time as well as frequency diversity, which assists in mitigating the detrimental effects of PBNJ. Furthermore, in the FFH-MFSK receiver, suitable diversity combining method may be invoked to further suppress the effects of PBNJ. We employ clipped combining [1] for this purpose.

Although Soft Decision Decoding (SDD) of noncoherent MFSK based schemes have been investigated in published literature [2]-[5], coded FFH schemes employing SDD have attracted little attention. By contrast, a number of useful tools have been employed for analysis as well as for performance enhancement of iteratively decoded coherently modulated schemes [6]-[8]. A powerful technique of enhancing the iterative gain is constituted by precoders, which improves the Extrinsic Information (EI) exchange between the channel decoder and the demodulator [8]. The precoder imposes memory upon the channel, thus rendering it recursive. ${ }^{1}$ Hence, SoftInput-Soft-Output (SISO) systems have been shown to benefit

\footnotetext{
The financial support of the EPSRC, UK and EU under the auspices of the Phoenix and Newcom projects and of Higher Education Commission, Pakistan is gratefully acknowledged.

${ }^{1}$ Recursivity in this context implies that the channel has an infinite impulse response.
}

substantially from employment of unity-rate precoders without reducing the effective throughput of the system [8].

In analogy to Irregular Convolutional Code (IrCC) [6], the novel family of so-called Irregular Variable Length Codes (IrVLC) [9] comprises of a number of component VLC codebooks having different coding rates [10] for encoding particular fractions of the input source symbol stream. With the aid of EXtrinsic Information Transfer (EXIT) charts [7], the appropriate lengths of these fractions may be chosen in order to shape the inverted EXIT curve of the IrVLC codec to ensure that it does not cross the EXIT curve of the inner channel decoder. In this way, an open EXIT chart tunnel may be created even at low Signal-to-Noise Ratio (SNR) values.

In [11], we proposed serial concatenation of a FFH-MFSK demodualor, a unity-rate decoder and an IrVLC decoder and investigated the 2-stage EI exchange between the unity-rate decoder and an IrVLC decoder. In this contribution we extend this concept to the 3 -stage EI exchange amongst the demodulator, inner decoder and the outer decoder. By employing EXIT charts, we investigate the serial concatenation of the FFHMFSK demodulator, unity rate decoder and the IrVLC outer decoder in order to attain a good performance even at low SNR values. We contrast the 2-stage Iterative Decoding (ID) between the inner rate-1 decoder and the IrVLC outer decoder to the 3-stage scheme and demonstrate that the 3-stage scheme outperforms the 2-stage scheme.

The rest of this paper is structured as follows. In Sec. II, the system considered is described and the concept of joint source and channel coding is elaborated. In Sec. III, the soft metrics are derived and the ID process is discussed with emphasis on the EXIT characteristics. In Sec. IV, the choice of IrVLC code parameters is discussed with the aid of EXIT charts and our Bit Error Ratio (BER) results are discussed. Finally, in Sec. V, we present our conclusions.

\section{System OVERVIEW}

In this section we consider an IrVLC codec and an equivalent regular VLC-based bench-marker in this role. We refer to these schemes as the IrVLC- and the VLC-FFH-MFSK arrangements, respectively. The schematic that is common to both of these schemes is shown in Fig. 1.

Joint source and channel coding We consider $K=16$-ary source symbol values that have the probabilities of occurrence that result from the Lloyd-Max (LM) quantization [11], [12] 


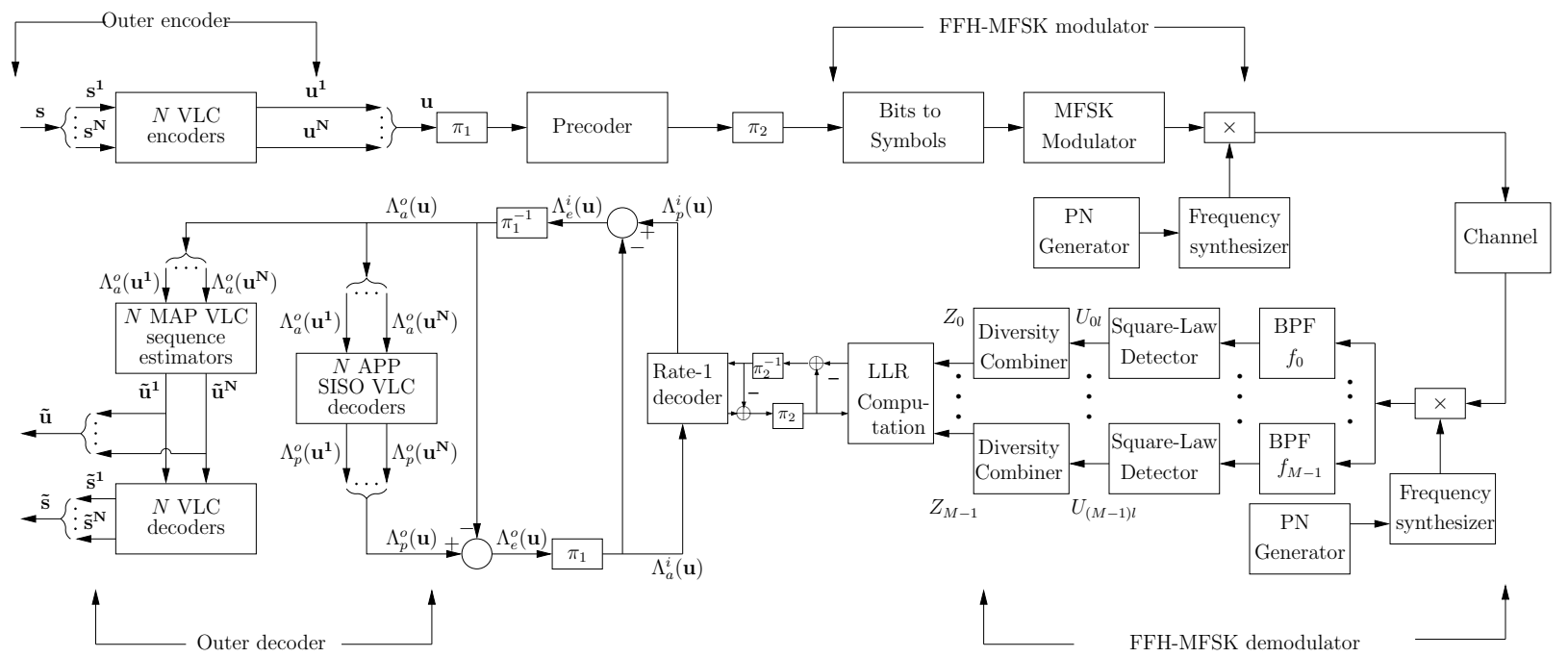

Fig. 1. Schematic of the IrVLC- and VLC-based schemes, employing 3-stage serial concatenation of the demodulator, rate-1 decoder and outer VLC/IrVLC decoder. In the IrVLC coded scheme $N=16$, whilst $N=1$ in the VLC-coded scheme.

of independent Laplacian distributed source samples. More explicitly, we consider the 4-bit LM quantization of a Gaussian source. Note that these occurrence probabilities vary by more than an order of magnitude between 0.0023 and 0.1616 . These probabilities correspond to entropy or average information values between 2.63 bits and 8.74 bits, motivating the application of VLC and giving an overall source entropy of $E=3.47 \mathrm{bits} / \mathrm{VLC}$ symbol.

In the transmitter of Fig. 1, the source symbol frame $\mathrm{s}$ to be transmitted is partitioned into number $J$ of 4-bit source symbols corresponding to $K=16$-ary values of $\left\{s_{j}\right\}_{j=1}^{J} \in$ $[1 \ldots K]$. These 4-bit source symbols are then decomposed into number $N$ of component streams $\left\{\mathbf{s}^{\mathbf{n}}\right\}_{n=1}^{N}$ to be protected by the $N$ different rate IrVLC component codes, where we opted for $N=16$ in the case of the IrVLC-FFH-MFSK scheme and $N=1$ in the case of the VLC-based benchmarker scheme. The number of symbols in the source symbol frame $\mathrm{s}$ that are decomposed into the source symbol frame component $\mathbf{s}^{\mathbf{n}}$ is specified as $J^{n}$, where we have $J^{1}=J$ in the case of the VLC-based scheme. By contrast, in the case of the IrVLCbased scheme, the specific values of $\left\{J^{n}\right\}_{n=1}^{N}$ may be chosen in order to shape the inverted EXIT curve of the IrVLC codec so that it does not cross the EXIT curve of the inner decoder, as detailed in Sec. IV.

Each of the $N$ source symbol frame components $\left\{\mathbf{s}^{\mathbf{n}}\right\}_{n=1}^{N}$ is VLC-encoded using the corresponding codebook from the set of $N$ VLC codebooks $\left\{\mathbf{V L C}^{n}\right\}_{n=1}^{N}$, having a range of coding rates $\left\{R^{n}\right\}_{n=1}^{N} \in[0,1]$, satisfying $\sum_{n=1}^{N} \alpha_{n} R^{n}=R$, where $\alpha_{n}=J^{n} / J$ is the particular fraction of the source symbol frame coded by the $n$th sub-code and $R$ is the average code rate of the VLC or the IrVLC scheme. The specific source symbols having the value of $k \in[1 \ldots K]$ and encoded by the specific VLC codebook VLC ${ }^{n}$ are represented by the codeword VLC ${ }^{n, k}$, which has a length of $I^{n, k}$ bits. The $J^{n}$ VLC codewords that represent the $J^{n}$ source symbols in the source symbol frame component $\mathbf{s}^{\mathbf{n}}$ are concatenated to provide the transmission frame component $\mathbf{u}^{\mathbf{n}}=\left\{\mathbf{V L C} \mathbf{C}^{n, s_{j^{n}}^{n}}\right\}_{j^{n}=1}^{J^{n}}$.

Owing to the variable length of the VLC codewords, the number of bits comprised by each transmission frame component $\mathbf{u}^{\mathbf{n}}$ will typically vary from frame to frame. In order to facilitate the VLC decoding of each transmission frame component $\mathbf{u}^{\mathbf{n}}$, it is necessary to explicitly convey the exact number of bits $I^{n}=\sum_{j^{n}=1}^{J^{n}} I^{n, s_{j^{n}}^{n}}$ to the receiver with the aid of side information. Furthermore, this highly errorsensitive side information must be reliably protected against transmission errors. This may be achieved using a low rate block code or repetition code, for example. For the sake of avoiding obfuscating details, this is not shown in Fig. 1.

FFH-MFSK modulation: The $N$ transmission frame components $\left\{\mathbf{u}^{\mathbf{n}}\right\}_{n=1}^{N}$ encoded by the different IrVLC component codes are concatenated at the transmitter, as shown in Fig. 1. The resultant transmission frame $\mathbf{u}$ has a length of $\sum_{n=1}^{N} I^{n}$ bits. Following the bit interleaver $\pi_{1}$, the binary transmission frame $\mathbf{u}$ is precoded and then interleaved again by the bit interleaver $\pi_{2}$. The precoded and interleaved bits are converted to $M$-ary symbols [4], which are transmitted by the FFHMFSK modulator of Fig. 1. To elaborate, in the FFH-MFSK transmitter the $M$-ary symbols are mapped to $M$ frequency tones [13]. The particular MFSK tone chosen for transmission modulates a carrier generated by a frequency synthesizer, which is controlled by the $L$-tuple FFH sequence generated by a pseudo-noise $(\mathrm{PN})$ generator, where $L$ is the number of frequency hops per symbol [1], [13].

The Channel: The channel is assumed to be a frequencyflat Rayleigh fading medium for each of the transmitted frequencies. Furthermore, we assume that the separation between the adjacent frequencies, which also equals the bandwidth occupied by a single FFH-MFSK tone $R_{h}=1 / T_{h}$, is higher than the coherence bandwidth of the channel. Therefore, all FFH tones conveying the same symbol experience independent 
fading.

The transmitted signal is also corrupted by Additive White Gaussian Noise (AWGN) and a PBNJ signal [13] having single-sided power spectral densities of $N_{0}$ and $N_{J}$, respectively. We assume that the PBNJ signal jams a fraction $0 \leq$ $\rho \leq 1$ of the total spread spectrum bandwidth $W_{s s}$. We also assume that the PBNJ signal is contiguous and hence all the $M$ FSK tones of a particular band are jammed, if the jamming signal is present in that band. Thus, the probability that a band or a tone is jammed is given by $\rho$, while the probability that the band is not jammed is $(1-\rho)$.

FFH-MFSK demodulation: The receiver schematic is also shown in Fig. 1, where the frequency de-hopper, which is identical to and aligned with the frequency hopper of the transmitter, de-spreads the received signal by exploiting the knowledge of the transmitter's unique FFH address. The demodulator of Fig. 1 is comprised of $M$ branches, each corresponding to a single MFSK tone and consisting of a bandpass filter (BPF), a square-law detector [13] as well as a diversity combiner, which performs clipping followed by linear combining of the signals received in all hops. The process of clipping is expressed by [1], [14]

$$
\begin{aligned}
f\left(U_{m l}\right)= & \begin{cases}C, & \text { if } U_{m l} \geq C \\
U_{m l}, & \text { otherwise }\end{cases} \\
& m=0,1, \ldots, M-1, l=0,1, \ldots, L-1,
\end{aligned}
$$

where $U_{m l}$ represents the square-law detector's output seen in Fig. 1 corresponding to the $m$ th tone in the $l$ th hop and $C$ represents an appropriately chosen clipping threshold. The decision variable recorded after clipped combining is given by

$$
Z_{m}=\sum_{l=0}^{L-1} f\left(U_{m l}\right), \quad m=0,1, \ldots, M-1 .
$$

At the output of the diversity combiner, the corresponding symbol probabilities and Log-Likelihood Ratios (LLRs) are computed, as explained in the following section, which are then fed to the unity-rate decoder of Fig. 1.

\section{ITERATIVE DECODING}

In this section we discuss how soft information is derived from the channel's output observations and how ID is carried out by exchanging EI between the demodulator, the unity-rate decoder and the outer decoder seen in Fig. 1.

Derivation of soft information In order to compute the LLRs, we need the probability that the $m t h$ symbol $s_{m}$ was transmitted, $m=0, \ldots, M-1$, given that the signal $\mathbf{Z}=$ $\left[Z_{0}, Z_{1}, \ldots, Z_{M-1}\right]$, which represents the set of $M$ outputs of the diversity combiners of Fig. 1, is received. This probability is given by [3], [14]

$$
P\left(s_{m} \mid \mathbf{Z}\right)=\frac{p\left(\mathbf{Z} \mid s_{m}\right) P\left(s_{m}\right)}{p(\mathbf{Z})},
$$

where $p\left(\mathbf{Z} \mid s_{m}\right)$ is the Probability Density Function (PDF) of the received signal $\mathbf{Z}$, given that $s_{m}$ is transmitted. Furthermore, $P\left(s_{m}\right)$ is the a priori probability of the symbol $s_{m}$, while $p(\mathbf{Z})=\sum_{m=0}^{M-1} p\left(\mathbf{Z} \mid s_{m}\right) P\left(s_{m}\right)$ is the probability of receiving signal set $\mathbf{Z}$, which is a constant for a given Z. Moreover, for equiprobable symbols, we have $P\left(s_{m}\right)=$ $1 / M$. Hence, the PDF $p\left(\mathbf{Z} \mid s_{m}\right)$ uniquely and unambiguously describes the statistics required for estimating the probability $P\left(s_{m} \mid \mathbf{Z}\right)$. For independent fading of all tones, the PDF $p\left(\mathbf{Z} \mid s_{m}\right)$ is given by [3], [14]

$$
p\left(\mathbf{Z} \mid s_{m}\right)=f_{Z_{m}}\left(x_{m} \mid s_{m}\right) \prod_{n=0, n \neq m}^{M-1} f_{Z_{n}}\left(x_{n} \mid s_{m}\right),
$$

where $f_{Z_{n}}\left(x_{n} \mid s_{m}\right)$ represents the PDF of the $n$th diversity combiner output, $n=0,1, \ldots, M-1$, given that $s_{m}$ is transmitted. In [14], using the PDFs and the Characteristic functions [13] of the square-law detector outputs, we derived a simplified expression for the $p\left(\mathbf{Z} \mid s_{m}\right)$ of (4) given by

$$
p\left(\mathbf{Z} \mid s_{m}\right)=\exp \left(\frac{x_{m} \gamma_{h}}{1+\gamma_{h}}\right),
$$

where $\gamma_{h}=b R E_{b} /\left(N_{0} L\right)$ is the SNR per hop, $E_{b}$ is the transmitted energy per bit and $b=\log _{2} M$ is the number of bits per MFSK symbol. Upon inserting (5) in (3), we can derive the corresponding symbol probabilities. The resultant bit probabilities can be derived from the symbol probabilities, assuming the bits-to-symbol mapping of [4] and finally, using the bit probablities, the LLRs can be computed [7].

Note that above, we have derived the soft information from the channel's output observations, assuming a somewhat simplistic but tractable interference-free channel. Moreover, although clipped combining is employed in the receiver, our analysis assumes linear combining, using no clipping at the receiver. This assumption has been stipulated for further simplifying the analysis and is supported by the observation that clipping is an operation performed to reduce effects of PBNJ. We will demonstrate in Sec. IV, that valuable performance improvements can be achieved using this sub-optimal soft information.

Three-stage concatenated scheme: Following our derivation of the soft information from the received signal, two types of serial concatenation schemes are possible. In the first case, the a posteriori probablity (APP) SISO unity-rate decoder and the outer decoder perform ID, both invoking the BCJR algorithm using bit-based trellises [11], [15]. We refer to this configuration of the ID as the 2-stage scheme. Alternatively, the system may be modified so that the FFHMFSK demodulator, the unity-rate inner decoder and the IrVLC outer decoder exchange their EI, as shown in Fig. 1. We refer to this arrangement as the 3-stage scheme. The 3stage scheme requires an additional interleaver $\pi_{2}$ between the precoder and the FFH-MFSK modulator of Fig. 1.

EXIT chart analysis: Let us now study the EI characteristics of the ID process using EXIT curves [7]. All EI measurements were made using the histogram method based approximation of the true distribution [7]. Note that in Fig. 1, $\Lambda(\cdot)$ denotes the LLRs of the bits concerned, where the superscript $i$ indicates inner decoder (or demodulator), while 


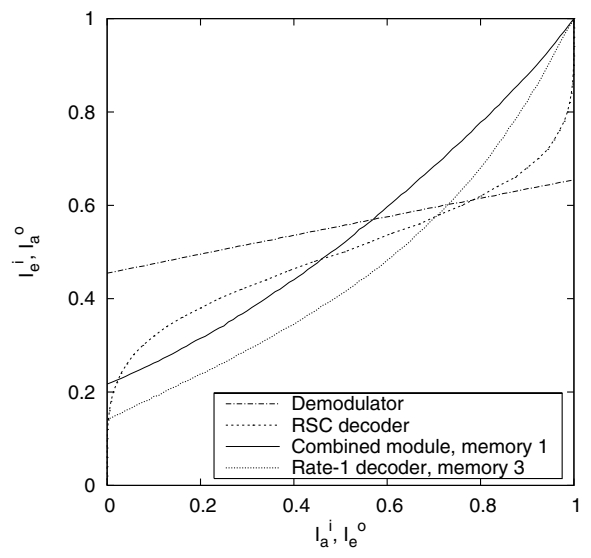

Fig. 2. EXIT characteristics of the demodulator, the rate-1 decoder and the combined module in Rayleigh fading channel contaminated by PBNJ, assuming $E_{b} / N_{0}=6 \mathrm{~dB}, E_{b} / N_{J}=10 \mathrm{~dB}$, and $\rho=0.1$.

$o$ corresponds to outer decoding. Additionally, a subscript denotes the dedicated role of the LLRs, with $a, p$ and $e$ indicating a priori, a posteriori and extrinsic information, respectively. Moreover, unless otherwise stated, we employ the following parameter values: source symbol frame length $J=70000$, code rate $R=0.5$, MFSK modulation order of $M=16$ and FFH diversity order of $L=3$. Finally, we assume optimum clipping thresholds, defined in (1), for all simulations.

In [11] we demonstrated that the FFH-MFSK demodulator yields low-gradient EXIT curves for all values of the modulation order $M$. When the SNR is sufficiently high, the EXIT curves can be shifted upward in the EXIT plane and hence an arbitrarily low BER may be achieved. However, this would be achieved at the cost of having a large area between the demodulator's and the decoder's EXIT curves, implying that the scheme operates far from capacity.

By contrast, it was shown in [11] that the precoder renders the channel to appear recursive [8], and hence has steeper EXIT curves than the stand-alone demodulator. Furthermore, as the precoder's memory is increased, the EXIT curves become steeper. Moreover, in contrast to the demodulator, the rate-1 decoder's EXIT curves do indeed reach the $\left(I_{e}, I_{a}\right)=$ $(1,1)$ point, implying that the precoder allows the ID to converge to an arbitrarily low BER.

Let us now employ the EXIT charts in order to investigate the 3-stage scheme. Ideally, this would require a 3-D EXIT chart depicting the evolution of the EI at the output of all the three serially concatenated components. However, a simpler and almost equally effective method of investigating this 3way EI exchange was proposed in [16], which implies treating the demodulator and the unity-rate inner decoder as a single module. This effectively allows us to analyze the 3-stage concatenation as a 2-stage one using 2-D EXIT charts. We will refer to this module as the combined module.

In Fig. 2, we compare the EXIT characteristics of the three types of inner modules considered, namely the stand-alone FFH-MFSK demodulator, the stand-alone rate- 1 decoder and the combined module in a channel contaminated by PBNJ. In this figure, we have assumed $E_{b} / N_{0}=6 \mathrm{~dB}, E_{b} / N_{J}=10 \mathrm{~dB}$ and a jamming duty factor of $\rho=0.1$. In order to portray these EXIT curves in the correct perspective, the inverted EXIT curve of a half-rate Recursive Systematic Convolutional (RSC) decoder characterised by the octal generator polynomial of $(7,5)$ is also shown. We observe that the combined module has superior EXIT curve and although its EXIT curve has similar gradient as that of the rate- 1 decoder, its EXIT curve emerges from a higher point in the EXIT plane, indicating that the combined module would allow satisfactory communication at lower SNR values. The superiority of the combined module over the stand-alone rate- 1 decoder stems from the fact that when employing the combined module, EI is also exchanged between the two SISO modules, i.e. the demodulator and the rate-1 decoder, thus enabling optimum exploitation of the soft information.

Note that our combined module invokes a single iteration of EI exchange between the demodulator and the unity-rate decoder. We have also investigated the use of more than one iterations between these two blocks but it was found that no additional benefit is achieved. Hence, when the 3-stage scheme of Fig. 1 is employed, each ID iteration involves one iteration between the demodulator and the unity-rate decoder followed by one iteration between the the unity-rate inner decoder and the outer decoder. Moreover, for the 3-stage scheme we employed a precoder of memory 1, while for the 2-stage scheme we employed a precoder of memory 3 , since it was discovered that using these precoder memories attractive EXIT characteristics are achieved.

VLC decoding: Since $N$ separate VLC encoders are employed in the IrVLC-FFH-MFSK transmitter, $N$ separate VLC decoders are employed in the corresponding receiver seen in Fig. 1. In parallel to the composition of the bit-based transmission frame $\mathbf{u}$ from $N$ VLC components, the a priori LLRs $\Lambda_{a}^{o}(\mathbf{u})$ are decomposed into $N$ components, as shown in Fig. 1. This is achieved with the aid of the explicit side information that we assumed for conveying the number of bits $I^{n}$ in each transmission frame component $\mathbf{u}^{\mathbf{n}}$. Each of the $N$ VLC decoders is provided with the a priori LLR subframe $\Lambda_{a}^{o}\left(\mathbf{u}^{\mathbf{n}}\right)$ and in response it generates the a posteriori LLR sub-frame $\Lambda_{p}^{o}\left(\mathbf{u}^{\mathbf{n}}\right), n \in[1 \ldots N]$. These a posteriori LLR sub-frames are concatenated in order to provide the $a$ posteriori LLR frame $\Lambda_{p}^{o}(\mathbf{u})$, as shown in Fig. 1 .

During the final decoding iteration, $N$ bit-based MAP VLC sequence estimation processes are invoked instead of singleclass APP SISO VLC decoding, as shown in Fig. 1. In this case, each transmission frame component $\mathbf{u}^{\mathbf{n}}$ is estimated from the corresponding a priori LLR frame component $\Lambda_{a}^{o}\left(\mathbf{u}^{\mathbf{n}}\right)$. The resultant transmission frame component estimates $\tilde{\mathbf{u}}^{\mathbf{n}}$ of Fig. 1 may be concatenated to provide the transmission frame estimate $\tilde{\mathbf{u}}$. Additionally, the transmission frame component estimates $\tilde{\mathbf{u}}^{\mathbf{n}}$ may be VLC decoded to provide the source symbol frame component estimates $\tilde{\mathbf{s}}^{\mathbf{n}}$. The interested reader may refer to [11] for a more detailed discussion on the decoding process. 


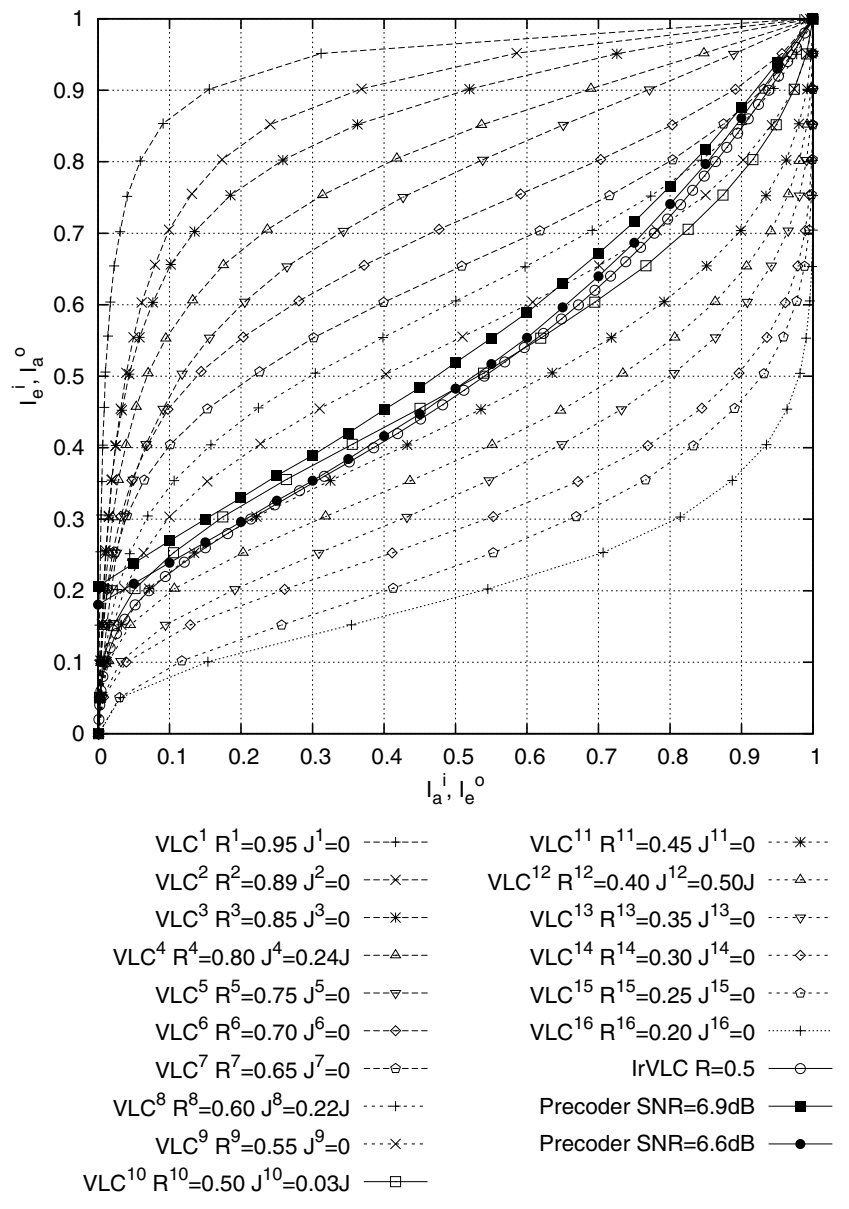

Fig. 3. Inverted VLC EXIT curves and rate-1 decoder EXIT curves, assuming $E_{b} / N_{J}=10 \mathrm{~dB}$, and $\rho=0.1$ in uncorrelated Rayleigh fading channel.

\section{SYSTEM PARAMETER DESIGN AND RESULTS}

As described in Sec. II, we opted for employing $N=16$ component VLC codebooks $\left\{\mathbf{V L C}^{n}\right\}_{n=1}^{N}$ having approximately equally spaced coding rates in the range of $[0.2,0.95]$ in the IrVLC-FFH-MFSK scheme of Fig. 1. In each case, we employ a Variable Length Error Correcting (VLEC) codebook [10] that is tailored to the source symbol values' probabilities of occurrence described in Sec. II. By contrast, in the VLC scheme, we employ just $N=1$ VLC codebook, which is identical to the VLC codebook VLC ${ }^{10}$ of the IrVLC scheme, having a coding rate of $R=0.5$. Note that this coding rate results in an average interleaver length of $J \cdot E / R$ bits.

In Fig. 3 we provide the inverted EXIT curves that characterise the bit-based APP SISO VLC decoding of the abovementioned VLC codebooks, together with the rate-1 decoder's EXIT curves at $E_{b} / N_{0}$ values of 6.6 and $6.9 \mathrm{~dB}$, assuming an uncorrelated Rayleigh fading channel contaminated by PBNJ characterised by $E_{b} / N_{J}=10 \mathrm{~dB}$ and $\rho=0.1$. All the EXIT curves were generated using uncorrelated Gaussian distributed a priori LLRs, based on the assumption that the transmission frame's bits have equiprobable logical values. This is justified because we employ a long interleaver length and because the entropy of the VLC encoded bits was found to be only negligibly different from unity for all the VLC codebooks considered.

Fig. 3 also shows the inverted EXIT curve of the IrVLC scheme. This was obtained as the appropriately weighted superposition of the $N=16$ component VLC codebooks' inverted EXIT curves, where the weight applied to the inverted EXIT curve of the component VLC codebook $\mathrm{VLC}^{\mathbf{n}}$ is proportional to the specific number of source symbols employed for encoding $J^{n}$ [6]. Using the approach of [6], the values of $\left\{J^{n}\right\}_{n=1}^{N}$ given in Fig. 3 were designed so that the IrVLC coding rate matches that of our regular VLC scheme, namely 0.5. Furthermore, we ensured that the inverted IrVLC EXIT curve did not cross the rate-1 decoder's EXIT curve at $E_{b} / N_{0}$ of $6.6 \mathrm{~dB}$. We note that only 4 of the 16 VLC components were indeed activated by the algorithm of [6] in order to encode a non-zero number of source symbols. As shown in Fig. 3, the presence of the resultant open EXIT chart tunnel implies that an infinitesimally low BER may be achieved by the IrVLC-FFH-MFSK scheme for $E_{b} / N_{0}$ values above $6.6 \mathrm{~dB}$. By contrast, an open EXIT chart tunnel is not afforded for $E_{b} / N_{0}$ values below $6.9 \mathrm{~dB}$ in the case of the benchmarker VLC-based scheme. Analogous to the IrVLC design of Fig. 3, we have also designed IrVLC codes for both the 2-stage and 3 -stage ID scheme, assuming various jamming scenarios in Rayleigh fading channels.

Let us now focus our attention on the BER performance of the proposed system as well as on that of the benchmarker in the context of the 2-stage and the 3-stage schemes considered. Note that the BER depicted in Figs. 4 to 5 corresponds to the encoded bits of the transmission frame $\mathbf{u}$ and the corresponding received frame $\tilde{\mathbf{u}}$, seen in Fig. 1 .

In Fig. 4 , we provide the BER versus $E_{b} / N_{J}$ performance comparison of both the 2-stage and the 3-stage schemes, assuming $E_{b} / N_{0}=10 \mathrm{~dB}$ and $\rho=0.5$. We observe that both the VLC and the IrVLC-based schemes result in superior performance compared to the system operating without the precoder, which encounters an error-floor. We also note that the 3-stage IrVLC scheme yields a further improvement of nearly $3 \mathrm{~dB}$ over the 2-stage IrVLC. This performance gain confirms the EXIT chart prediction of Fig. 2. However, the performance gain achieved by employing 3-stage concatenation comes at the cost of an increased complexity, since an additional interleaver is required in the 3-stage scheme as shown in Fig. 1. Moreover, each decoding iteration of the 3 -stage scheme involves the additional EI exchange with the demodulator as well. Finally, we note in Fig. 4 that the IrVLC based 3-stage scheme outperforms the corresponding VLCbased scheme by approximately $1.1 \mathrm{~dB}$.

An increased complexity is also imposed by the increased number of decoding iterations, which is a natural consequence of operating at lower SNR. This is especially true in case of the IrVLC scheme, where typically a narrower EXIT tunnel exists between the EXIT curves of the outer and the inner decoders, as seen in Fig. 3. In Fig 4, up to 80 iterations are needed for achieving convergence to the $\left(I_{e}, I_{a}\right)=(1,1)$ 


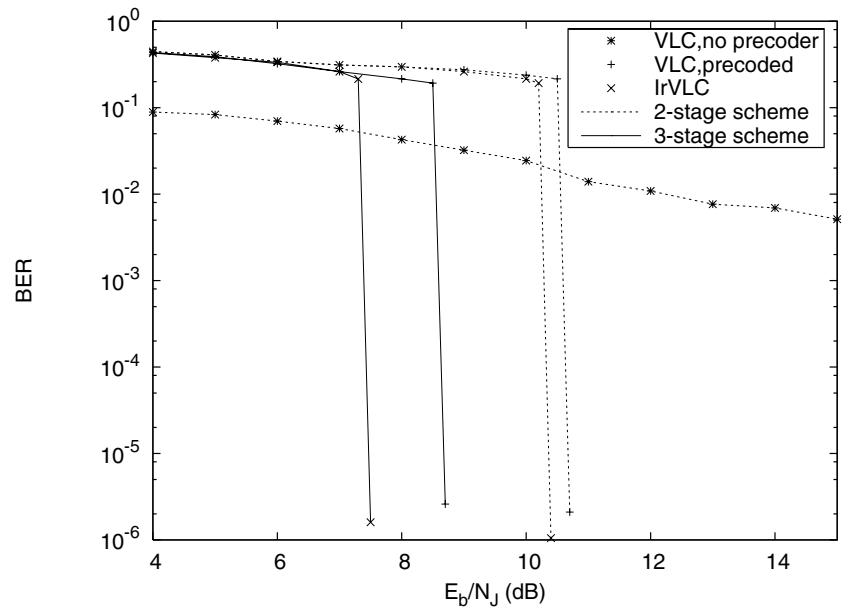

Fig. 4. BER versus $E_{b} / N_{J}$ performance of the 2-stage and 3-stage VLC and IrVLC based schemes in jammed, uncorrelated Rayleigh fading channels, assuming $E_{b} / N_{0}=10 \mathrm{~dB}$ and $\rho=0.5$.

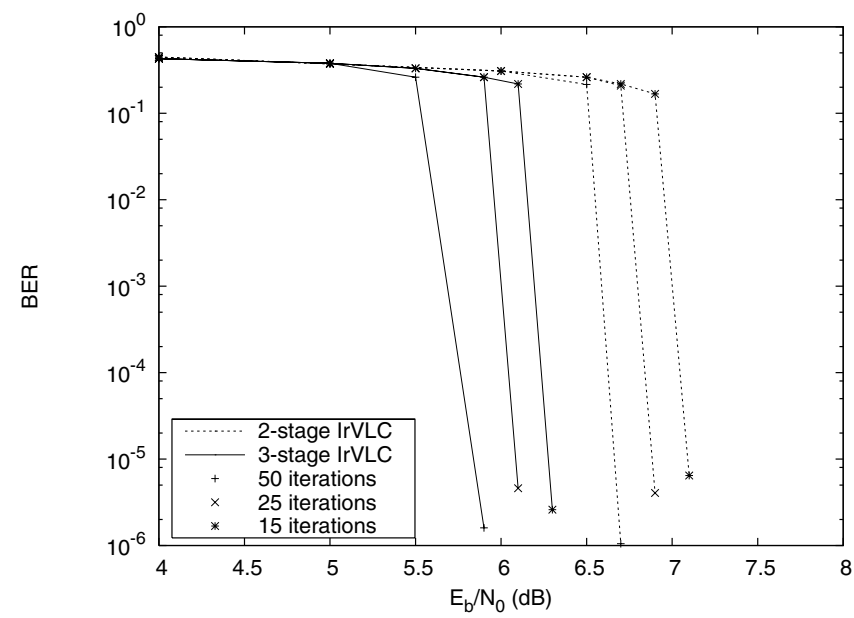

Fig. 5. BER versus $E_{b} / N_{0}$ performance of the 2-stage and 3-stage IrVLC based schemes in jammed, uncorrelated Rayleigh fading channels for various number of decoding iterations, assuming $E_{b} / N_{J}=10 \mathrm{~dB}$ and $\rho=0.1$.

when employing IrVLC-based scheme. The effect of reducing the number of iterations on both the 2-stage and the 3-stage schemes is depicted in Fig. 5, where the BER versus $E_{b} / N_{0}$ performance of the IrVLC schemes considered is shown. We observe that the number of decoding iterations can be significantly reduced by raising the $E_{b} / N_{0}$ value by as little as 0.2 to $0.4 \mathrm{~dB}$.

\section{CONCLUSION}

We have investigated the serial concatenation of IrvLC coding with a FFH-MFSK modem operating in a Rayleigh fading channel, when the transmitted signal was corrupted by PBNJ. We employed 2-D EXIT charts to investigate the 3stage concatenation of the FFH-MFSK demodulator, the rate1 decoder and the outer IrVLC decoder. Consequently we noted that the precoder-aided schemes yield a $E_{b} / N_{0}$ gain in excess of $7 \mathrm{~dB}$ over the system dispensing with the precoder, which suffers from an error floor when jamming is severe.
The IrVLC code was designed in such a way that the inverted EXIT curve of the IrVLC decoder matches the EXIT curve of the inner decoder. In this way, an open EXIT chart tunnel may be created even at low SNR values, providing sourcecorrelation-dependent additional performance gains of up to $1.1 \mathrm{~dB}$ over the VLC-based scheme.

Moreover, we demonstrated that the 3-stage concatenation scheme yields superior performance compared to the 2-stage concatenation, yielding a gain of nearly $3 \mathrm{~dB}$.

\section{REFERENCES}

[1] K. C. Teh, A. C. Kot, and K. H. Li, "FFT-based clipper receiver for fast frequency-hopping spread-spectrum system," in Proceedings of the IEEE Symposium on Circuits and Systems, vol. 4, pp. 305-308, MayJune 1998.

[2] P. C. P. Liang and W. E. Stark, "Algorithm for joint decoding of turbo codes and M-ary orthogonal modulation," in IEEE International Symposium on Information Theory, 2000, p. 191, June 2000.

[3] M. C. Valenti, E. Hueffmeier, B. Bogusch, and J. Fryer, "Towards the capacity of noncoherent orthogonal modulation: BICM-ID for turbo coded NFSK," in IEEE Conference on Military Communications, 2004. MILCOM 2004, vol. 3, pp. 1549 - 1555, October-November 2004.

[4] U. C. Fiebig, "Soft-decision and erasure decoding in fast frequencyhopping systems with convolutional, turbo, and Reed-Solomon codes," IEEE Transactions on Communications, vol. 47, pp. 1646 - 1654, November 1999.

[5] D. Park and B. G. Lee, "Iterative decoding in convolutionally and turbo coded MFSK/FH-SSMA systems," in IEEE International Conference on Communications, ICC 2001, vol. 9, pp. 2784 - 2788, June 2001.

[6] M. Tüchler and J. Hagenauer, "EXIT charts of irregular codes," in Conference on Information Sciences and Systems, (Princeton, NJ), pp. 748-753, March 2002.

[7] S. ten Brink, "Convergence of iterative decoding," IEEE Transactions on Communications, vol. 49, pp. 1727 - 1737, October 2001.

[8] R. Y. S. Tee, S. X. Ng, and L. Hanzo, "Precoder-aided iterative detection assisted multilevel coding and three-dimensional EXIT-chart analysis," in IEEE Wireless Communications and Networking Conference, 2006. WCNC 2006, vol. 3, pp. 1322-1326, April 2006.

[9] R. G. Maunder, J. Wang, S. X. Ng, L.-L. Yang, and L. Hanzo, "Iteratively Decoded Irregular Variable Length Coding and Trellis Coded Modulation," in IEEE Workshop on Signal Processing Systems, (Shanghai, China), October 2007.

[10] V. Buttigieg and P. G. Farrell, "Variable-length error-correcting codes," IEE Proceedings on Communications, vol. 147, pp. 211-215, August 2000.

[11] S. Ahmed, R. G. Maunder, L. L. Yang, S. X. Ng, and L. Hanzo, "Joint Source Coding, Unity Rate Precoding and FFH-MFSK Modulation using Iteratively Decoded Irregular Variable Length Coding," in Proceedings of the 66th IEEE Vehicular Technology Conference, 2007. VTC Fall, 2007, pp. 1042-1046, Sep-Oct 2007.

[12] S. Lloyd, "Least squares quantization in PCM," IEEE Transactions on Information Theory, vol. 28, no. 2, pp. 129-137, 1982.

[13] J. G. Proakis, Digital communications. Singapore: Mcgraw-Hill, 2001.

[14] S. Ahmed, S. X. Ng, L. L. Yang, and L. Hanzo, "Iterative Decoding and Soft Interference Cancellation in Fast Frequency Hopping Multiuser System Using Clipped Combining," in Proceedings of the IEEE Wireless Communications and Networking Conference, WCNC 2007., pp. 723 728, March 2007.

[15] V. B. Balakirsky, "Joint source-channel coding with variable length codes," in IEEE International Symposium on Information Theory, (Ulm, Germany), p. 419, June 1997.

[16] J. Wang, S. X. Ng, A. Wolfgang, L.-L. Yang, S. Chen, and L. Hanzo, "Near-capacity three-stage MMSE turbo equalization using irregular convolutional codes," in International Symposium on Turbo Codes, (Electronic publication, Munich Germany), April 2006. 